\title{
Echocardiographic flow pattern of patent ductus arteriosus: a guide to indomethacin treatment in premature infants
}

\author{
Bai-Horng Su, Ching-Tien Peng, Chang-Hai Tsai
}

\begin{abstract}
Aim-To compare the efficacy and safety of an indomethacin treatment strategy based on serial echocardiographic measurement of patent ductus arteriosus (PDA) flow pattern with a standard protocol.

Methods-Neonates weighing less than $1500 \mathrm{~g}$ at birth, who required respiratory support, and who had developed symptomatic PDA, were studied. PDA was confirmed in all infants using colour Doppler echocardiography, and serial observations of the ductal flow pattern were made. Infants randomly assigned to receive conventional indomethacin treatment (protocol group) were given an initial dose of 0.2 $\mathrm{mg} / \mathrm{kg}$, followed by 0.1 or $0.2 \mathrm{mg} / \mathrm{kg}$, depending on age, 12 hourly for two further doses, and were eligible for a second course. Those randomly assigned to the ductal flow pattern assessment (ECHO group) received further doses of indomethacin after 24 hours, only if their flow pattern was "pulsatile" or "growing." Results-There was no significant difference in the primary outcome measures between the two groups. The closure rate was $89.1 \%$ and $87.2 \%$, respectively, in the protocol and ECHO groups. The mean (SD) doses of indomethacin were significantly higher in the protocol group: 3.2 (1.4) doses compared with $1.6(0.9)$ doses. There was a significantly higher incidence of hypoglycaemia, impaired urine output, and gastrointestinal bleeding in the protocol group.
\end{abstract}

Conclusions-An indomethacin treatment strategy for PDA based on measurement of the ductal flow pattern is associated with a reduction in the total doses of indomethacin administered, and a reduced rate of complications, compared with a conventional protocol. There is no difference in closure rate.

(Arch Dis Child Fetal Neonatal Ed 1999;81:F197-F200)

Department of

Paediatrics

China Medical College

Hospital

2 Yuh Der Road

Taichung

Taiwan

B-H Su

C-T Peng

C-H Tsai

Correspondence to: Dr Bai-Horng Su.

Accepted 15 June 1999 incomplete indomethacin course because of complications or contraindications following the first doses of indomethacin, but in whom the ductus eventually closed. On the other hand, closure failures have been observed in infants who received a complete or even repeat courses of indomethacin treatment.

Ductal tissues are sensitive to the dilating effects of prostaglandins, ${ }^{34}$ and increased concentrations of prostaglandins have been associated with ductal patency in premature infants..$^{5-7}$ However, a recent report by Gonzalez et al revealed that the increased risk of failed closure of PDA in infants with infection was not associated with an incomplete course of, or the absence of, indomethacin. ${ }^{8}$ This suggests that a complete course of indomethacin might not be warranted if the ductus can be closed after the first or second dose of indomethacin. This led us to explore which indicator could be used to determine the need for additional doses of indomethacin in infants. Using pulsed Doppler echocardiography, we have already reported that there is an association between pulsatile and growing patterns of PDA shunt flow and the development of clinically significant PDA. ${ }^{9}$

This study aimed to test the reliability of the assessment of PDA shunt flow pattern as a guide to the need for indomethacin treatment. We hypothesised that pulsatile and growing patterns might indicate the need for indomethacin treatment, and that the closing and closed patterns indicate that subsequent indomethacin doses could be withheld.

\section{Methods}

The study was conducted at the Tokyo Metropolitan Tsukiji Maternity Hospital, Japan, from August 1995 to December 1996, and at the China Medical College Hospital, Taichung, Taiwan, from February to October 1997. Premature infants weighing less than $1500 \mathrm{~g}$ at birth, who required assisted ventilation, and had a symptomatic PDA, were enrolled. General guidelines for the treatment of very low birthweight infants were similar in Tokyo and in Taichung. The fluid supply was $60-70$ $\mathrm{ml} / \mathrm{kg}$ on the first day, increasing by $10 \mathrm{ml} / \mathrm{kg}$ daily, and adjusted to maintain serum electrolytes within the normal range, until intake reached $120 \mathrm{ml} / \mathrm{kg}$ within the first week of life. Respiratory distress syndrome (RDS) was diagnosed according to clinical and chest radiographic findings consistent with RDS and requirements for inspired oxygen of $>40 \%$ and for mechanical ventilation. The surfactant 

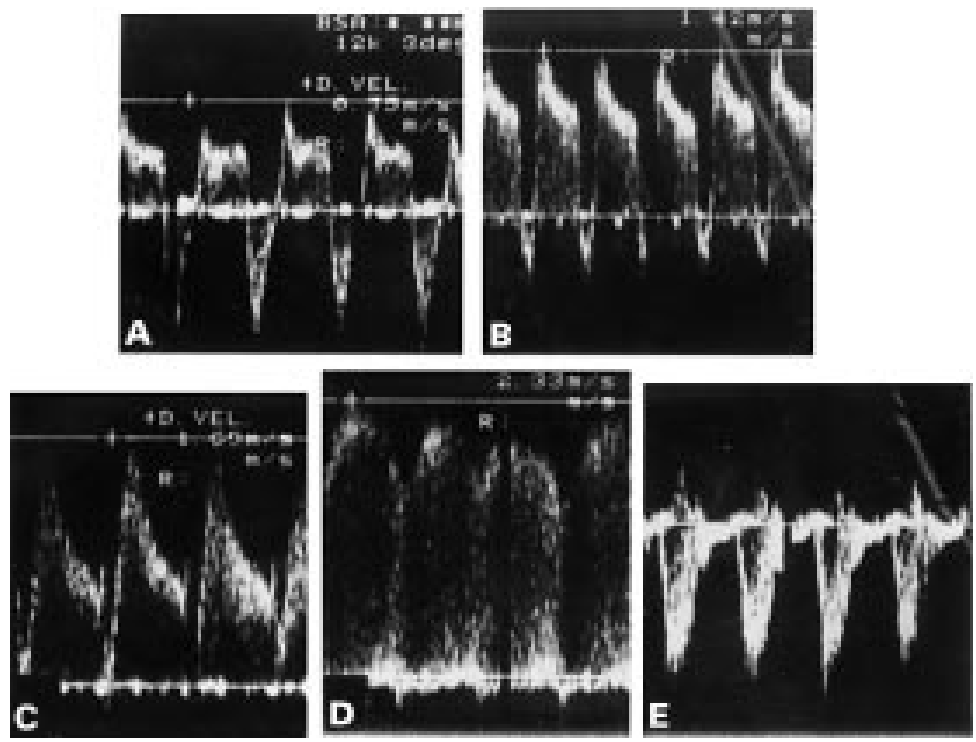

Figure 1 Doppler flow patterns: (A) pulmonary hypertension pattern; (B) growing pattern; (C) pulsatile pattern; (D) closing pattern; (E) closed pattern.

preparations used were Surfacten TA (Surfacten, Tokyo-Tanabe Company, Japan) in Tokyo and Survanta (Beractant, Abbott Laboratories, Chicago, USA) in Taichung. The dosing schedule was 1 phial Surfacten TA or $4 \mathrm{ml}$ Survanta per kg of body weight. Assisted ventilation was adjusted to maintain partial arterial oxygen tension $\left(\mathrm{PaO}_{2}\right)$ between 50 and 80 $\mathrm{mmHg}$, partial arterial carbon dioxide tension $\left(\mathrm{PaCO}_{2}\right)$ between 40 and $50 \mathrm{mmHg}$, and $\mathrm{pH}$ between 7.25 and 7.45 .

Symptomatic PDA was diagnosed when there was colour Doppler echocardiographic evidence of left to right ductal shunt associated with at least two of the following clinical signs: heart murmur; persistent tachycardia (heart rate $>160 / \mathrm{min}$ ); hyperactive precordial pulsation; bounding pulses; and radiographic evidence of cardiomegaly or pulmonary congestion. The patients were randomly assigned to receive indomethacin treatment according to the usual protocol (protocol group) or to echocardiographic PDA flow pattern (ECHO group). ${ }^{9}$ Excluded from the study were infants with congenital anomalies, congenital infections, and those who died during the first 72 hours of birth. The eligible infants not included during the study period were those admitted when the investigator with echocardiographic skills (BHS) was on leave, or when serial echocardiographic examination could not be performed.

The study protocol was approved by the review boards of both hospitals, and informed consent was obtained from each infant's parents.

ECHOCARDIOGRAPHIC DATA COLLECTION

In Tokyo, the infants were studied using a Hewlett-Packard 77020A or an Aloka SSD2000 scanner fitted with a probe which incorporated a $5 \mathrm{MHZ}$ colour and pulsed Doppler transducer. The first echocardiographic study confirmed cardiac normality and the ductus arteriosus was imaged from the high left parasternal view using colour Doppler echocardiography. If PDA was present, the pulsed Doppler gate was placed in the pulmonary end of the ductus, taking care to ensure the angle of insonation was below 20 degrees, and the flow pattern of the shunt was recorded.

The PDA flow was classified as follows: (1) pulmonary hypertension pattern, which has a bi-directional flow profile with a predominant right to left shunt (downward away from the baseline); (2) growing pattern, which also has a bi-directional flow but with a predominant left to right shunt (upward away from the baseline); (3) pulsatile pattern, which has a left to right shunt with a pulsatile profile of peak flow velocity about $1.5 \mathrm{~m} / \mathrm{second}$; and (4) closing pattern, which also has a left to right shunt, but with a characteristic continuous profile of peak flow velocity of about $2 \mathrm{~m} / \mathrm{second}$ (fig 1 ).

In Taichung the infants were studied using an Acouson $128 \mathrm{XP} / 10$ scanner with 5 or 7 MHZ colour and pulsed Doppler transducer. The ductus arteriosus was imaged as in Tokyo.

INDOMETHACIN TREATMENT FOR PATENT

DUCTUS ARTERIOSUS

Protocol group

Colour Doppler echocardiographic examination was performed daily 12-24 hours after birth until the ductus arteriosus closed. Infants were examined at least twice daily for clinical signs of PDA, and diagnosis and treatment protocols were made by the attending clinician. The management protocol for a symptomatic PDA in both units included fluid restriction and intravenous administration of indomethacin $(0.2 \mathrm{mg} / \mathrm{kg}$ for the first dose, then 0.1 $\mathrm{mg} / \mathrm{kg}$ in infants less than 48 hours old, 0.2 $\mathrm{mg} / \mathrm{kg}$ in infants over 48 hours, every 12 hours for another two doses) if not contraindicated. $^{910}$ A follow up echocardiogram was obtained between 24 to 36 hours after completion of the course of indomethacin treatment. If the PDA remained open, as documented on colour Doppler echocardiography, a second course of indomethacin was given and the echocardiogram was repeated 24 to 36 hours afterwards, and then every 24 hours until closure. If the PDA had not closed and the patient remained symptomatic after the second course of indomethacin, surgical ligation was considered. Surgery was also performed in infants with symptomatic PDA in whom indomethacin was contraindicated.

\section{ECHO group}

Infants in this group also received colour Doppler echocardiographic and clinical assessments. Similarly, indomethacin was given when a symptomatic PDA was diagnosed. Pulsed Doppler echocardiography was performed immediately before indomethacin treatment to determine the PDA flow pattern. A follow up echocardiogram was obtained 24 hours after the start of the first dose of indomethacin. The echocardiographer (BHS) was blinded to the clinical status of the infants. PDA flow pattern and the treatment regimen were decided by the attending clinician. Subsequent doses of indomethacin were given only if the pulsatile or growing PDA flow pattern persisted on follow 
Table 1 Demographic data of infants

\begin{tabular}{llll}
\hline & $\begin{array}{l}\text { Protocol group } \\
(n=46)\end{array}$ & $\begin{array}{l}\text { ECHO group } \\
(n=47)\end{array}$ & $p$ Value \\
\hline Gestational age (weeks) & $27.8(2.5)$ & $27.2(2.6)$ & 0.277 \\
Birthweight (g) & $1039(244)$ & $955(271)$ & 0.09 \\
Sex (male/female) & $24 / 22$ & $21 / 26$ & 0.605 \\
Surfactant treatment (n \%) & $40(87 \%)$ & $42(89.4 \%)$ & 0.967
\end{tabular}

The values are mean (SD) or number of infants (percentage).

up pulsed Doppler echocardiograms obtained every 24 hours. The subsequent dose of indomethacin was withheld if the closing or closed pattern was documented. If a closing or closed pattern was not observed after six doses of indomethacin, surgical ligation was considered. Surgery was also considered in infants with symptomatic PDA in whom indomethacin was contraindicated.

OUTCOME MEASURES

General clinical data, total doses of indomethacin, PDA outcome, and the presence of complications associated with prematurity and indomethacin treatment, as well as neonatal mortality and the incidence of chronic lung disease were recorded. Complications associated with indomethacin occurring during treatment or within 48 hours of its completion, such as hypoglycaemia, impaired urine output, necrotising enterocolitis and gastrointestinal bleeding, were also recorded. Hypoglycaemia was diagnosed if blood glucose fell to below 40 $\mathrm{mg} / \mathrm{dl}$; impaired urine output was defined as a fall to less than half that before treatment started. Necrotising enterocolitis was considered if there were signs of abdominal distension and ileus, and was confirmed by a roentgenogram showing a fixed dilated loop, pneumatosis, portal or hepatic venous air or pneumoperitoneum. Gastrointestinal bleeding was considered if the aspirate had the appearance of coffee grounds, or if there was fresh blood from the gastric tube.

Cranial sonograms were obtained on days 1 , 7,28 , and, if necessary, when intraventricular haemorrhage (IVH) was suspected. IVH grading was performed according to Papile et al. ${ }^{11}$ PDA outcome was categorised as: (1) failure of closure, if the PDA remained open as documented on echocardiography for at least 10 days or if surgical ligation was required for symptomatic PDA, because of unresponsiveness or contraindication of indomethacin treatment, or (2) reopening of PDA, if there

Table 2 Clinical results after indomethacin treatment

\begin{tabular}{llll}
\hline & $\begin{array}{l}\text { Protocol group } \\
(n=46)\end{array}$ & $\begin{array}{l}\text { ECHO group } \\
(n=47)\end{array}$ & p Value \\
\hline Age at treatment (days) & $2.6(1.9)$ & $2.3(1.1)$ & 0.999 \\
Doses of indomethacin (number) & $3.2(1.4)$ & $1.6(0.9)$ & $<0.01$ \\
Closure rates & $41(89.1 \%)$ & $41(87.2 \%)$ & 0.999 \\
Reopening rates & $4(8.7 \%)$ & $5(10.6 \%)$ & 0.999 \\
Ligation rates & $5(10.9 \%)$ & $5(10.6 \%)$ & 0.999 \\
Complications & $21(45.7 \%)$ & $10(21.3 \%)$ & 0.02 \\
$\quad$ Hypoglycaemia & $4(8.7 \%)$ & $4(8.5 \%)$ & 0.999 \\
$\quad$ Necrotising enterocolitis & $26(56.5 \%)$ & $12(25.5 \%)$ & 0.0046 \\
$\quad$ Impaired urine output & $18(39.1 \%)$ & $8(17.0 \%)$ & 0.03 \\
$\quad$ Gastrointestinal bleeding & $9(19.6 \%)$ & $9(19.1 \%)$ & 0.999 \\
IVH (all grades) & $3(6.5 \%)$ & $3(6.4 \%)$ & 0.999 \\
IVH (grades 3 and 4) & $9(19.6 \%)$ & $9(19.2 \%)$ & 0.999 \\
Neonatal mortality & $9(24.3 \%)$ & $10(26.3 \%)$ & 0.999 \\
Chronic lung disease in survivors & & & \\
\hline
\end{tabular}

The values are mean (SD) or number of infants (percentage). was clinical and echocardiographic evidence of PDA after initial closure. Neonatal mortality was defined as death within 28 days of age. Chronic lung disease was defined as requirement for supplemental oxygen at 28 days of age, with respiratory distress and characteristic signs on chest $x$-ray picture.

STATISTICAL ANALYSIS

Our previous study showed that about half of the infants, similar to those enrolled in this study, had at least one of the major complications associated with indomethacin treatment. $^{1012}$ To examine whether PDA flow pattern is an effective guide to indomethacin treatment, we estimated that the sample should comprise 45 infants in each group, to achieve a power of $80 \%$ at a level of $p=0.05$. We expected a decrease in the complication rate associated with indomethacin treatment from $50 \%$ to $20 \%$, when PDA flow pattern was adopted as a guide to treatment. The observed PDA incidence was $60 \%$ in infants weighing under 1500 $\mathrm{g}$, therefore, a total of 150 infants were needed for the study. Categorical variables were analysed using Student's $t$ test, and the comparisons of complication rates were carried out using $\chi^{2}$ analysis.

\section{Results}

A total of 173 infants weighing less than 1500 $\mathrm{g}$ at birth, and who required assisted ventilation, were admitted during the study period. Of these, 11 died during the first 72 hours of life, 109 developed clinical signs of suspected PDA, and 10 (including two with congenital anomalies, one with congenital infection, and seven not confirmed by colour Doppler echocardiography) were excluded.

The remaining 99 infants had symptomatic PDA confirmed by echocardiography and received indomethacin. Of these, six did not receive follow up echocardiography. Therefore, 93 infants with symptomatic PDA were eligible for the final analysis; 46 in the protocol group, and 47 in the ECHO group. The demographic data of all infants are summarised in table 1 . There were no significant differences in mean gestational age, mean birthweight, sex distribution and percentage of surfactant treatment between the two groups.

The mean doses of indomethacin, the age at first dose, PDA outcomes and complication rates, as well as neonatal mortality and the incidence of chronic lung disease are summarised in table 2. There were no significant differences between the two groups in the age when given first indomethacin treatment, PDA closure rates, re-opening rates or ligation rates, neonatal mortality or the incidence of chronic lung disease among survivors. Among the major complications associated with indomethacin treatment, there was no significant difference in the incidence of necrotising enterocolitis, but significant differences were noted in the incidences of hypoglycaemia, impaired urine output, and gastrointestinal bleeding. There were no significant differences in the incidences of any grade of IVH or grades 3 and $4 \mathrm{IVH}$ between the two groups. 


\section{Discussion}

The overall closure rate of $88 \%(82 / 93)$ in this series is consistent with that of a previous report. ${ }^{13}$ Among the infants in whom PDA flow pattern was used as a guide to indomethacin treatment (ECHO group) and who did not complete the full course of three doses, the closure rate was $87.2 \%$. This study showed that indomethacin treatment guided by the PDA flow pattern is as good as our standard indomethacin treatment protocol in terms of PDA closure.

The introduction of surfactant replacement therapy means that PDA is diagnosed earlier and more frequently in premature infants. ${ }^{14-16}$ In our study $88 \%$ (82/93) infants received surfactant treatment. Our diagnosis of symptomatic PDA was made by combing through prospective daily echocardiography data and clinical findings.

A common indomethacin treatment strategy is one dose every 12 hours over a course of three doses. Clinically, there is a relatively high incidence of complications associated with indomethacin treatment, including hypoglycaemia, necrotising enterocolitis, decreased urine output and gastrointestinal haemorrhage. ${ }^{10}{ }^{12}$ A more selective policy of indomethacin treatment would be a more effective way of reducing complications. However, it is difficult to predict those infants who will not require indomethacin, those in whom the duct will close after one or two doses of indomethacin, and those who will fail to respond to the full course of treatment. Colour Doppler echocardiography, on the other hand, is a useful tool to assess the patency of ductus arteriosus and the direction of PDA shunt flow. Actual size of shunt flow and haemodynamic changes are, however, difficult to measure. Methods for calculating the PDA shunt volume from both ventricular outputs to predict a clinically significant PDA are complicated. ${ }^{17}$

We classified PDA shunt flow into four patterns using pulsed Doppler echocardiography, and showed that serial changes in the pattern can be used to predict the risk of developing clinically significant PDA. ${ }^{9}$ Our previous study suggested that the pulmonary hypertension pattern is common in early postnatal life, and reflects a bi-directional shunt, with only a little left to right shunting in the presence of a high pulmonary artery resistance. After the fall in pulmonary artery resistance, the left to right shunting through a constricted ductus produces a very rapid velocity, characterised by the closing pattern. In contrast, growing and pulsatile patterns are primarily evidence of falling pulmonary artery resistance, in the presence of a ductus which is not completely restricted and becomes clinically significant.

In this study we used PDA flow pattern analysis as a guide to indomethacin treatment, and this allowed us to use significantly fewer doses of indomethacin than normal, but without a higher incidence of closure failure. Furthermore, fewer doses were associated with a reduced rate of complications-hypoglycaemia, impaired urine output, and gastrointestinal bleeding. There was no obvious effect on neonatal morbidity or the incidence of chronic lung disease.

In conclusion, although PDA flow pattern assessment is a useful guide to the use of indomethacin treatment in infants with a PDA, it does require an operator who is skilled in the use of colour Doppler echocardiography. Moreover, the operator would need to be available on a daily basis, the implications of which have been discussed before. ${ }^{18}$

This study was supported by a grant from the Department of Medical Research, China Medical College Hospital (DMR-87Medical.

1 Friedman WF, Hirschklau MJ, Printz MP, Pitlick PT, Kirkpatrick SE. Pharmacological closure of patent ductus arteriosus in the premature infant. $N$ Engl $f \mathrm{Med}$ 1976;295:526-9.

2 Heymann MA, Rudolph AM, Silverman NH. Closure of the ductus arteriosus in premature infants by inhibition of prostaglandin synthesis. N Engl f Med 1976;295:530-3.

3 Coceani F, Olley PM. The response of the ductus arteriosus to prostaglantins. Can F Physiol Pharmacol 1973;51:220-5.

4 Clyman RI, Mauray F, Rudolph AM, Heymann MA. Age-dependent sensitivity of the lamb ductus arteriosus to indomethacin and prostaglandins. F Pediatr 1980;96:94-8.

5 Lucas M, Mitchell A. Plasma prostaglandins in preterm neonates before and after treatment for patent ductus arteriosus. Lancet 1978;ii:130-2.

6 Hutchison A, Ogletree M, Palme J, et al. Plasma 6-keto-prostaglantin F1a and thromboxane B2 in sick preterm neonates. Prostaglatins Leukotrienes and Medicine 1985;18:163-81.

7 Hammerman C, Zaia W, Berger S, et al. Prostaglatin levels: predictors of indomethacin responsiveness. Pediatr Cardiol 1986;7:61-5.

8 Gonzalez A, Sosenko IRS, Chandar J, Hummler H, Claure $\mathrm{N}$, Bancalari $\mathrm{E}$. Influence of infection on patent ductus
arteriosus and chronic lung disease in premature infants weighing 1000 grams or less. $\mathcal{F}$ Pediatr 1996;128:470-8.

9 Su BH, Watanabe T, Shimizu M, Yanagisawa M. Echocardiographic assessment of ductus arteriosus shunt flow pattern in premature infants. Arch Dis Child Fetal Neonatal Ed 1997;77:F36-F40.

10 Miyama N, Itoh R, Ohmori I, Su BH, Shimizu M, Watanabe T. Intravenous indomethacin treatment in very low birthweight infants with PDA: a retrospective study of 95 infants. Acta Neonat fpn 1995;31:854.

11 Papile LA, Burstein J, Burstein R, Koffler H. Incidence and evolution of subependymal and intraventricular hemmorrhage: a study of infants with birthweights less than 1500 rhage: a study of infants with

12 Su BH, Hu PS, Huang HW, Liu LY, Yang PH, Billiet M. A survey on the treatment strategy of patent ductus arteriosus in very low birthweight infants. Acta Paed Sin 1998;39:33-7.

13 Gersony WM, Peck GJ, Ellison RC, et al. Effects of indomethacin in premature infants with patent ductus arteriosus: results of a national collaborative study. $\mathcal{F}$ Pediatr 1983;102:895-906.

14 Kaapa P, Seppanen M, Kero P, Saraste M. Pulmonary hemodynamics after synthetic surfactant replacement in neonatal respiratory disease syndrome. $\mathcal{f}$ Pediatr 1993;123:115-9.

15 Reller MD, Buffkin DC, Colasurdo MA, Rice MT, McDonald RW. Ductal patency in neonates with respiratory disease syndrome: a randomized surfactant trial Am $\mathcal{F}$ Dis Child 1991;145:1017-20.

16 Reller MD, Rice MT, McDonald RW. Review of studies evaluating ductal patency in the preterm infant. $\mathcal{F}$ Pediatr 1993;122:S59-S62.

17 Evans NJ, Iyer P. Change in blood pressure after treatment of patent ductus arteriosus with indomethacine. Arch Dis Child 1993;68:584-7.

18 Skinner JR. Echocardiography on the neonatal unit: a job for the neonatologist of the cardiologist? Arch Dis Child 1998;78:401-2. 